Progress Report for DE-FG03-87ER 45331

August 10, 2003

\title{
MOLECULAR PROPERTIES OF SURFACES AND FILMS
}

\author{
Principal Investigator: Jacob Israelachvili \\ Ph. 1-805-893 8407, E-mail <jacob@engineering.ucsb.edu> \\ Dept Chemical Engineering, Materials Department, and Materials Research Laboratory, \\ University of California at Santa Barbara (UCSB), Santa Barbara, CA 93106.
}

Sect. No.

\section{THIS YEAR'S HIGHLIGHTS}

1. Characterizing 'normal' and 'inverted' stick-slip sliding

2. Tribology (and processing) of nanoparticle films

3. Friction of polymer-on-polymer surfaces

4. Adhesion and friction of rough (i.e., engineering) surfaces

5. Invited review article for Nature (on simplified theories of friction)

6. Measuring molecular organization in thin shearing interfacial films

7. Crude oil-surface-water interactions: reservoir wettability and oil-recovery

\section{REFERENCES TO DOE-FUNDED PAPERS (2003)}

[1] Friction between two weakly adhering boundary lubricated surfaces in water. Carlos Drummond, Jacob Israelachvili, Philippe Richetti. Phys. Rev. E 67 (6) (2003) 066110.

[2] Transitions between smooth and complex stick-slip sliding of surfaces. Delphine Gourdon and Jacob Israelachvili. Phys. Rev. E 68 (2003) 021602.

[3] Frictional forces and Amontons Law: from the molecular to the macroscopic scale. Jianping Gao, W. D. Luedtke, D. Gourdon, J. Israelachvili, U. Landman. J. Chem. Phys. 2003 (in press).

[4] Universality of the short-range exponential repulsion between rough particle surfaces. Jianping Gao, W. D. Luedtke, D. Gourdon, J. Israelachvili, U. Landman. J. Chem. Phys. (MS in preparation).

[5] Review article with M. Urbakh, J. Klafter, J. Israelachvili, D. Gourdon, for Nature.

[6] Fundamental studies of crude oil-surface-water interactions and its relationship to reservoir wettability. C. Drummond, J. Israelachvili. J. Pet. Sci. Eng. 2003 (in press).

\section{OTHER INFORMATION}

- Research planned for 2004

- Conference presentations

- Awards

- Personnel 


\section{Characterizing 'normal' and 'inverted' stick-slip sliding}

Stick-slip sliding and stiction spikes during a change in the sliding direction are important phenomena having both desirable and undesirable consequences. We have completed a detailed study of stick-slip friction, and especially the transitions between smooth and stick-slip sliding, of lubricated surfaces. Water-based lubricated systems (with boundary surfactant layers) were studied in Ref. [1], and oil-based lubricants in Ref. [2]. Both studies, which were carried out over a larger range of experimental conditions (of sliding velocity, applied load and temperatures) than in previous studies, revealed both lower and upper critical smooth-to-stick-slip sliding transitions. Ref. [1] also included a new theoretical treatment of these highly complex tribological systems that now appear to be the norm. Some of the more interesting findings are shown in Figs 1-4, taken from Ref. [1]. Others were described and shown in last year's report.
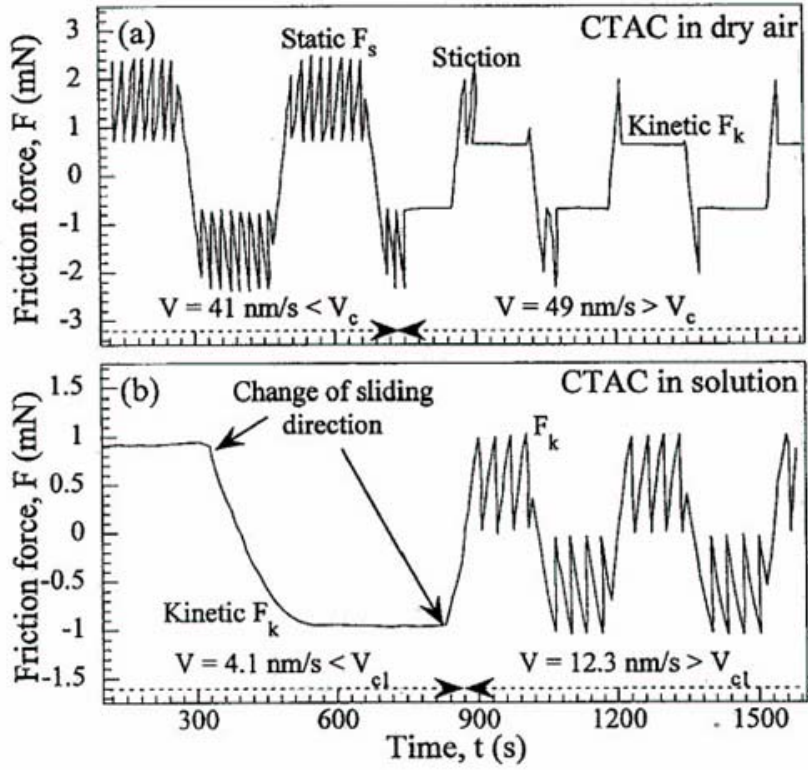

Fig. 1. Real friction traces (friction force versus time) showing stick-slip-to-smoothsliding transitions, and vice versa.

(a) Conventional stick-slip transition. The stick-slip regime occurs at low sliding velocities where the friction force oscillates between a high 'static' value $F_{\mathrm{s}}$ and a lower 'kinetic' value $F_{\mathrm{k}}$. Above the critical velocity $V_{c}$, the sliding is smooth (except for stiction).

(b) "Inverted" stick-slip. Smooth sliding now occurs at low velocities, $V<V_{c}$, and stickslip occurs above $V_{\mathrm{c}}$ where the friction force oscillates between the kinetic value $F_{k}$ and a lower value, $F_{\text {sk }}$. Note that in the smooth sliding regime, after a change in the shearing direction, there is no stiction spike [cf. (a)].

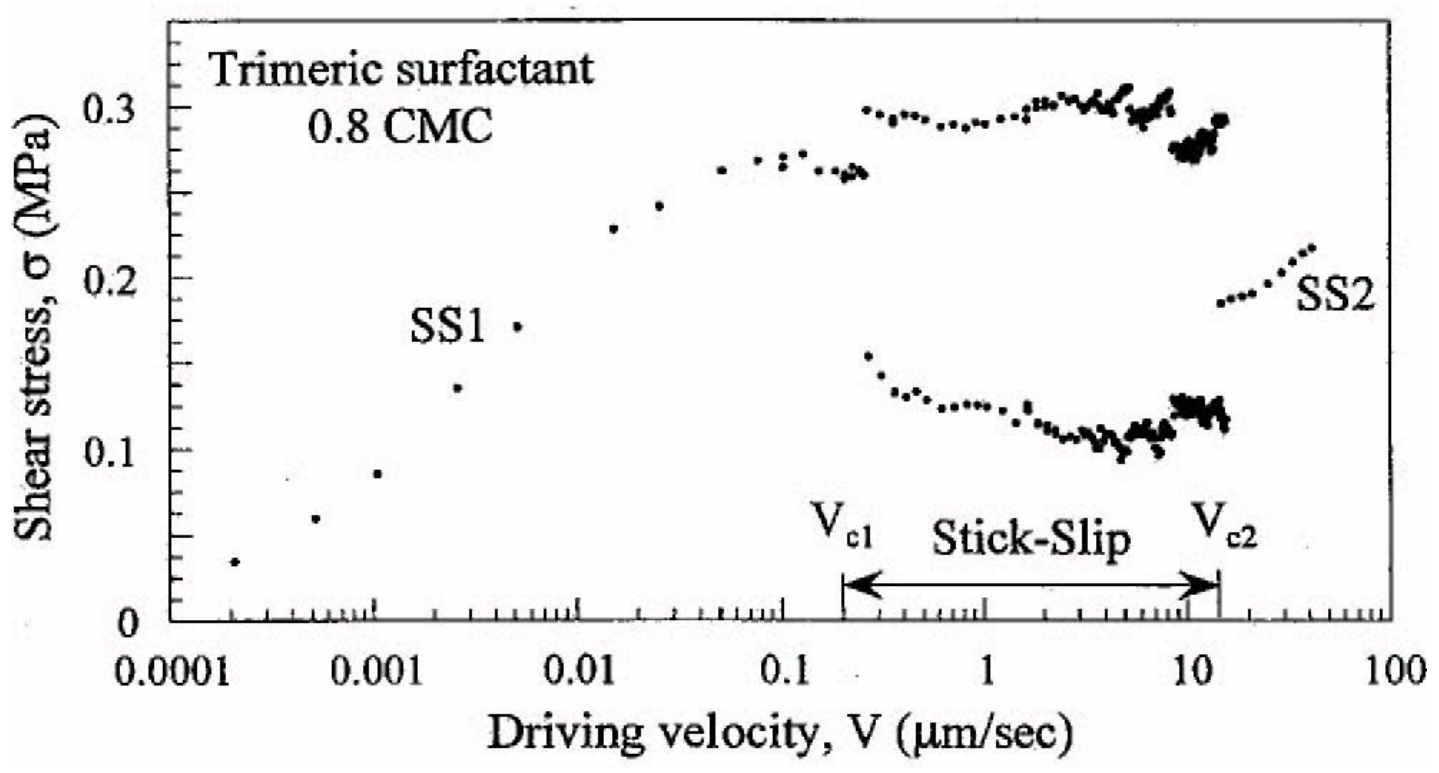


Fig. 2. Measured shear stress ( $\sigma=$ friction force/contact area) versus driving velocity $\mathrm{V}$ for two adsorbed positively charged surfactant monolayers on mica sliding in water under a fixed load. Smooth sliding (SS1) occurs at low V, and an 'inverted' smooth-to-stick-slip-sliding transition occurs at $V_{\mathrm{c} 1} \approx 0.3 \mu \mathrm{m} / \mathrm{s}$. Just prior to the transition, the stress $\sigma$ reaches a peak value. The stickslip regime is bounded by the high static and low kinetic values, as shown. At an even higher driving velocity, near $V_{\mathrm{c} 2} \approx 15 \mu \mathrm{m} / \mathrm{s}$, there is a 'normal' stick-slip-to-smooth-sliding transition.

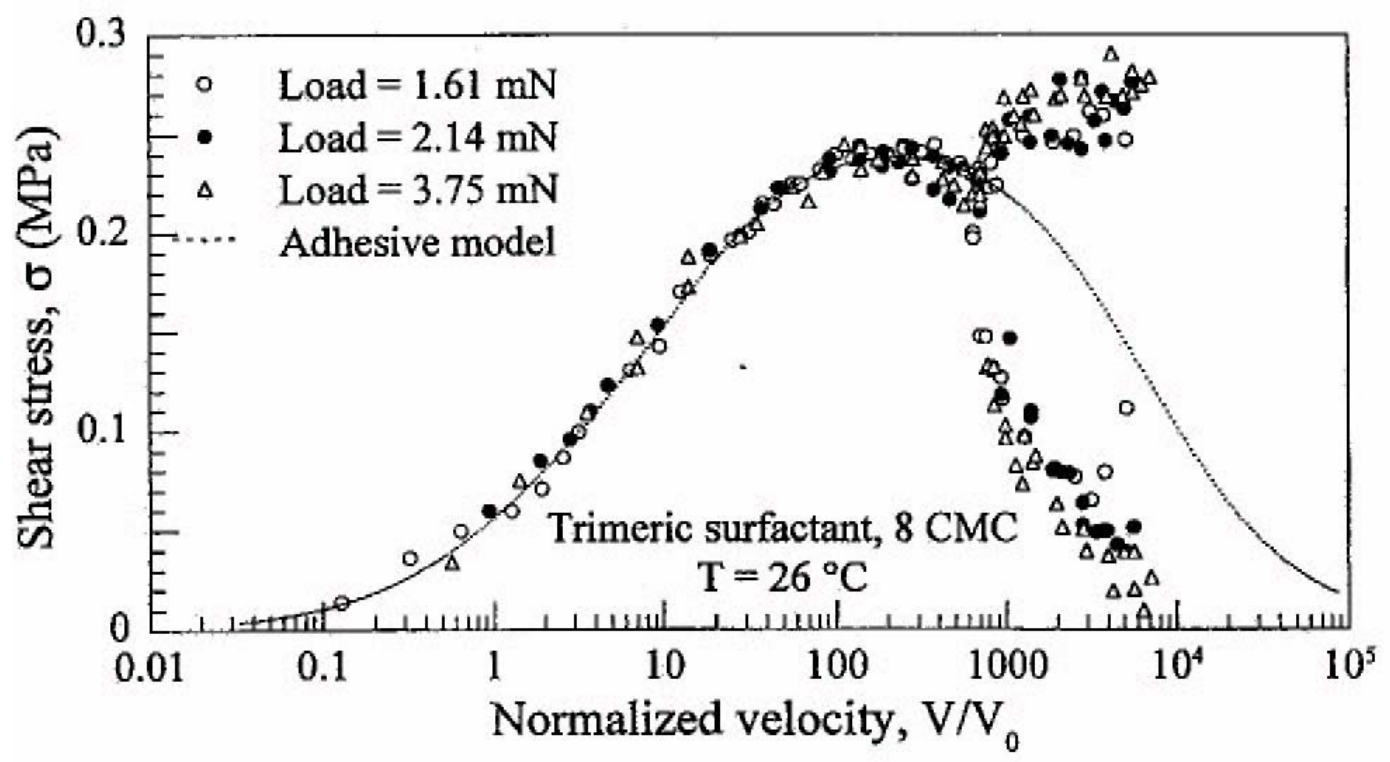

Fig. 3. Details of the 'inverted' smooth-to-stick-slip-sliding transitions shown in Fig. 2 at different normal loads, after normalizing the sliding velocity by $V_{\mathrm{c}}$. The dotted line is the theoretical fit obtained with the 'adhesive friction' model [1]. Experimentally, we expect stickslip to occur whenever $\mathrm{F}$ versus $\mathrm{V}$ has a negative slope, depending on the stiffness of the system.

\section{Tribology (and processing) of nanoparticle films}

In an attempt to understand the friction of nanoparticle films (which are often thought of as miniature ball bearings), we have sheared thin films of inorganic nanospheres and nano-rods (5-10 $\mathrm{nm}$ in diameter and 5-30 long) between two mica and surfactant-coated mica surfaces. To our surprise, some of our observations and conclusions go beyond the mere tribological characterization of these films. Thus, we find that compression and shear of nanoparticles of $\mathrm{BaCrO}_{4}$ and CdSe can order them in ways that cannot be done by other methods (see Figs 4 and 5).

Fig. 4. TEM images of a two-dimensional 'lattice' of $\mathrm{BaCrO}_{4}$ nanorods after compression and shear (see Fig. 5). Small angle $x$-ray diffraction spectrum of the confined nanorods show them to have an oblique cubic structure.

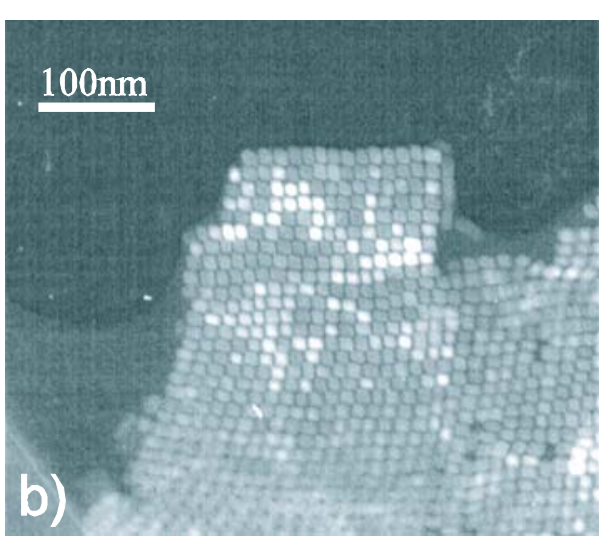




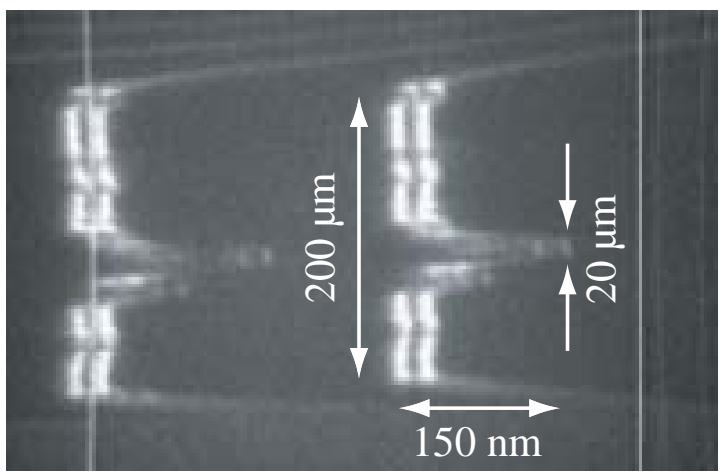

(a)

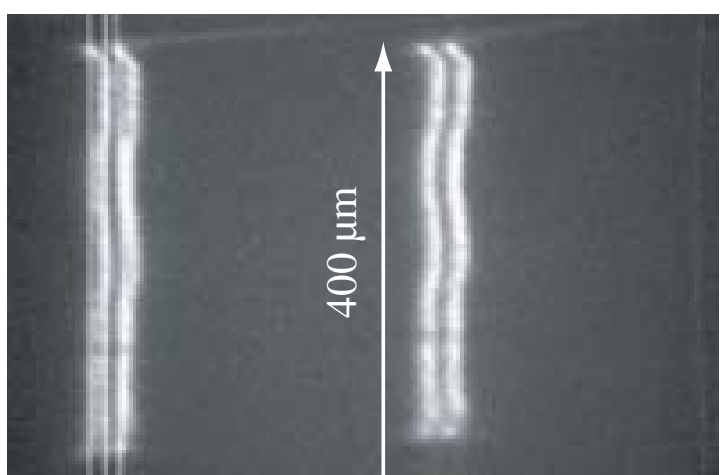

(b)

Fig. 5. FECO fringe images of confined $\mathrm{BaCrO}_{4}$ nanorod films (a) after being pressed together for the first time, showing some flattened regions (terraces) of roughly uniform thickness, but also heterogeneous clumps up to $150 \mathrm{~nm}$ high and $20 \mu \mathrm{m}$ wide. (b) At higher pressures (>40 MPa), and after some shear, the clumps have collapsed, leaving only one fairly uniform terrace extending over the whole contact area of diameter $>400$ $\mu \mathrm{m}$ and of thickness one, two or three nanorod diameters (10 $\mathrm{nm}$ in this case).

This new type of pressure and shear ordering is potentially important for processing thin crystalline films of nanoparticles that can have unique mechanical and especially opto-electronic properties. Preliminary results of our tribological studies of various nanoparticle films, including $\mathrm{BaCrO}_{4}$ and CdSe, are shown in Fig. 6.
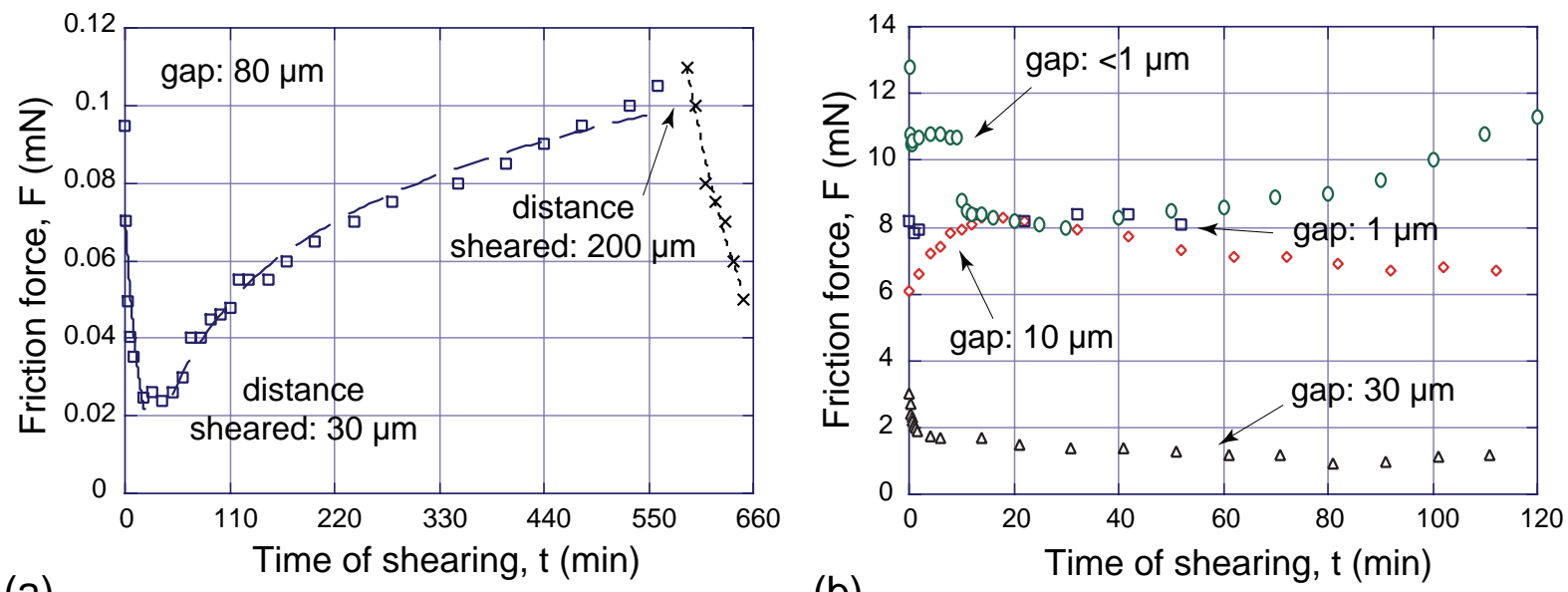

(a)

Fig. 6. (a) Friction force versus shearing time of a $80 \mu \mathrm{m}$ thick film of $\mathrm{BaCrO}_{4}$ nanorods. The shearing velocity was kept constant at $\mathrm{V}=5 \mu \mathrm{m} / \mathrm{s}$. At $\mathrm{t}=50 \mathrm{sec}$ the shearing amplitude (back and forth movement) was increased from 30 to $200 \mu \mathrm{m}$, and at $\mathrm{t}=600 \mathrm{sec}$ it was decreased again. It is clear that the shearing distance is important in determining the way a film adapts to its steadystate friction behavior [2]. (b) Effect of load (decreasing gap thickness) on the time-dependent friction force. As the load increases the friction increases roughly linearly, as expected; but there is less velocity or time-dependence for thinner films. The time-dependent tribological properties do not correlate simply with the structural changes, as they do for hydrocarbon films. 


\section{Friction of polymer-on-polymer surfaces}

We are following up on our recent work on the adhesion and friction mechanisms of polymeron-polymer surfaces, where our previous DOE-funded work [Maeda et al., Science 297 (2002) 379-382] suggested that the state of the outermost polymer segments determine their adhesion hysteresis (adhesion dynamics) and friction forces. We are studying polymers of varying molecular weight (chain length), degree of cross-linking, scission (free ends), polarity, layer thickness, both 'dry' and in liquid, or exposed to condensing vapors (Fig. 8); and we are also planning to use other experimental techniques, such as various spectroscopies, and x-ray and neutron scattering and reflectivity, to fully characterize these systems and the processes going on at the molecular level. Our results to date confirm that the last 8-10 segments are the most important, and that polarity plays a less significant role than the state of the polymer segments at the surfaces (i.e., whether linear, cross-linked, branched, etc.). These findings are consistent with our work on rough polymer surfaces (see Section 4 below) that also suggest that even the fine, atomic-scale structure of the surface molecular groups can have a large effect on the surface adhesion and friction.
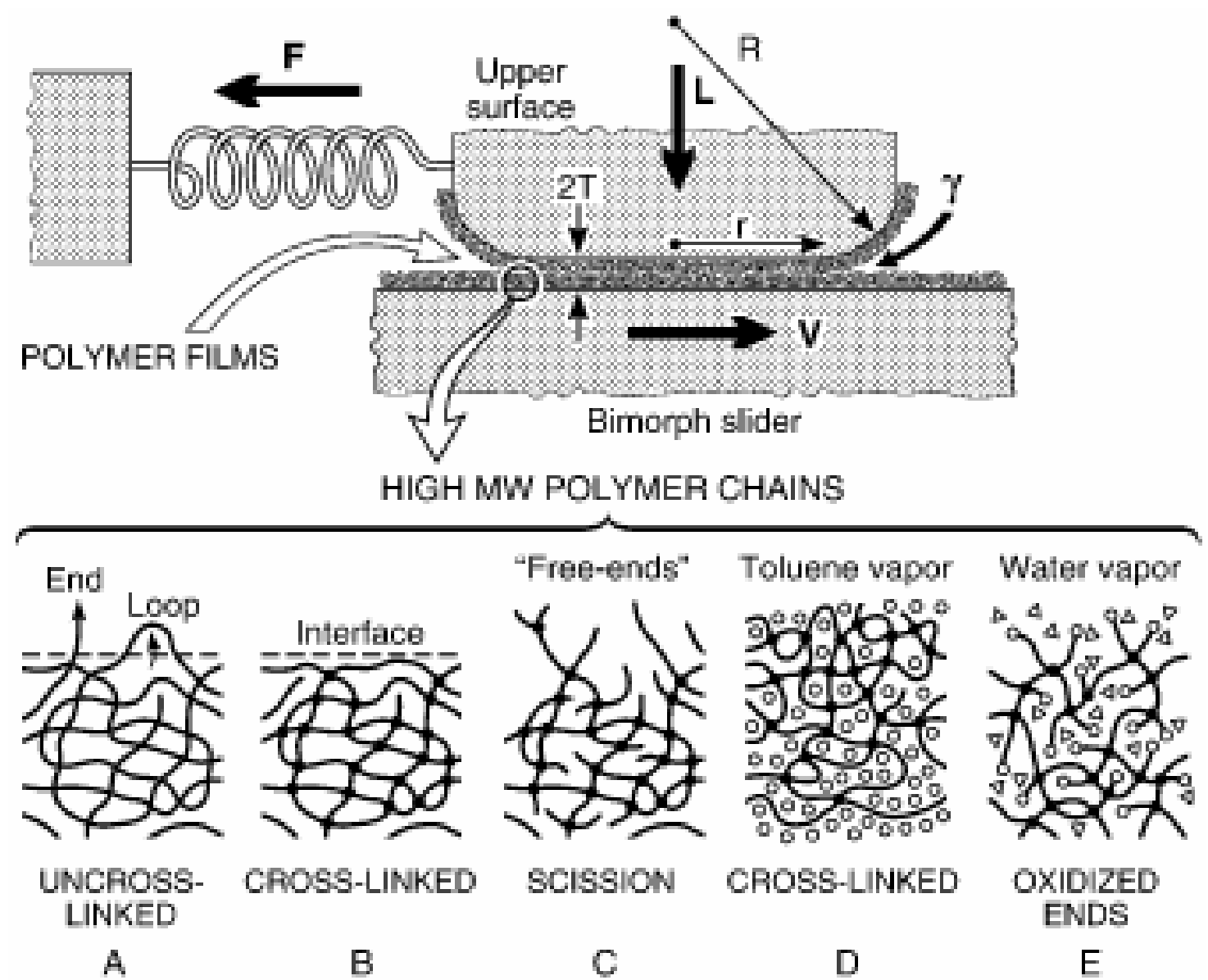

Fig. 7. Schematic of SFA friction measurements and what we are measuring with polymer surfaces under different conditions. 


\section{Adhesion and friction of rough (i.e., engineering) surfaces}

One of the main goals of this grant period is to investigate and quantify the role of surface roughness (which in the broader sense includes textured and patterned surfaces) on adhesion and friction. We first describe our recent friction experiments, where we have carried out a series of measurements on (nearly) identical surfaces using the SFA and AFM techniques, where the 'tip' radii $R$, contact areas, and contact pressures can differ by up to 6 orders of magnitude. The measured friction coefficients, defined by $\mu=$ friction force/applied load $=\mathrm{F} / \mathrm{L}$, were the same for both systems within 10\% (Fig. 8), and similar findings were obtained for other material surfaces (but of course with different values for $\mu$ ). While this is no more than a validation of Amontons' Law, its verification for such widely different geometries (length scales) and roughnesses suggests a universality that requires some theoretical explanation. Accordingly, with Dr Uzi Landman's group at Georgia Tech, we have recently completed a detailed analysis of a variety of different experimental results, and have written a lengthy article that will appear as a Review Article in J. Phys. Chem. [3].
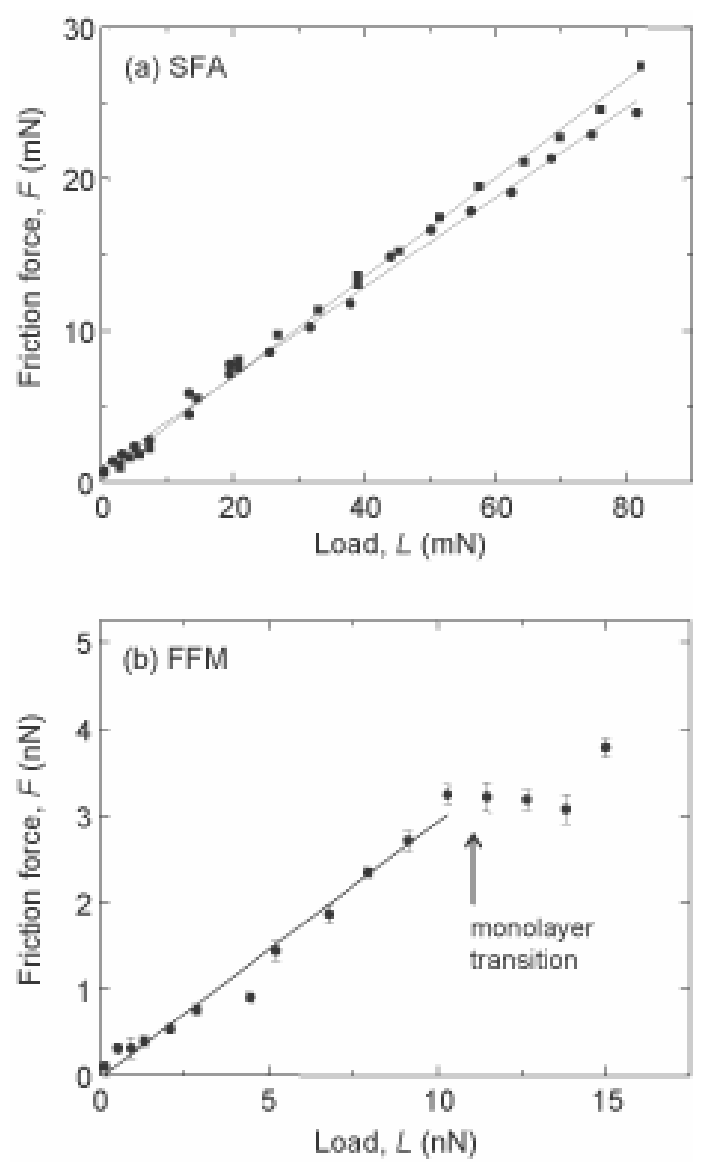

Fig. 8. Friction forces measured between benzyl-trichlorosilane monolayers chemically bound to glass or Silicon, in ethanol. (a) SFA measurements where both glass surfaces were covered with a monolayer. Circles and squares show two different experiments: one with $R=2.6 \mathrm{~cm}, V=0.15$ $\mu \mathrm{m} / \mathrm{s}$, giving $\mu=0.33 \pm 0.01$; the other with $R=1.6 \mathrm{~cm}, V=0.5 \mu \mathrm{m} / \mathrm{s}$, giving $\mu=0.30 \pm 0.01$. (b) AFM measurements of the same monolayer-functionalized Si tip $(R=11 \mathrm{~nm})$ sliding on a monolayer-covered glass surface at $V=0.15 \mu \mathrm{m} / \mathrm{s}$, giving $\mu=0.30 \pm 0.01$. 
In this article we first review the historical and modern understanding of Amontons Law - the most basic equation of friction - which states quite simply that the friction coefficient is constant and independent of the contact area, the surface roughness and the sliding velocity. No theory has yet satisfactorily explained this surprisingly general law, all attempts being model or system dependent. Molecular dynamics (MD) simulations were done on 'realistic' systems (Fig. 9), both adhesive and non-adhesive. The results are in excellent agreement with recent and past experiments, and with Amontons Law, and show that the local energy-dissipating mechanisms are not 'mechanical', as assumed in most models, but 'thermodynamic' - like miniature irreversible Carnot engines that cycle between compression and expansion as the surface asperities of two sliding surfaces past over each other. An interesting feature of the MD analysis is that for such dynamic, non-equilibrium, energy-dissipating processes, the Weibull distribution serves a similar purpose as the Boltzmann distribution for classical systems at equilibrium. Another interesting conclusion is that the concept of the 'area of contact' is an undefined, notfundamental and unnecessary quantity, whether at the nano-, micro- or macro-scales. However, it may serve as a convenient phenomenological parameter for describing the really fundamental parameters, which are the number or number density of atoms, molecules or bonds involved in an adhesive or frictional interaction.

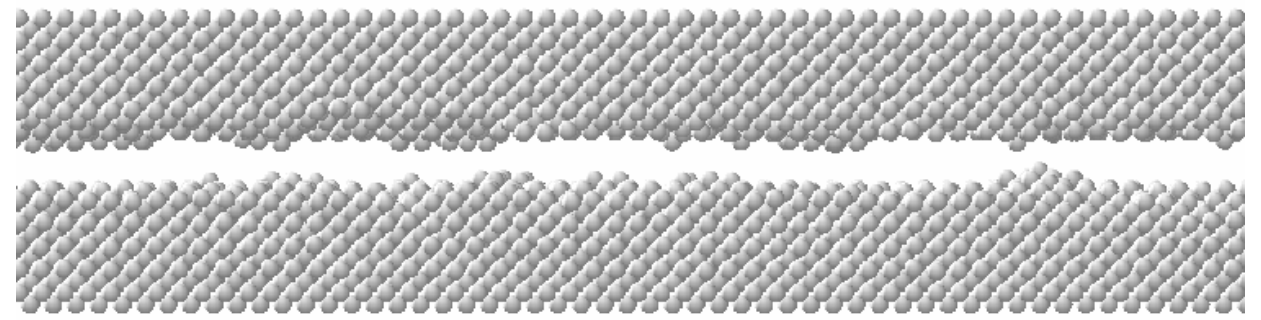

(a)

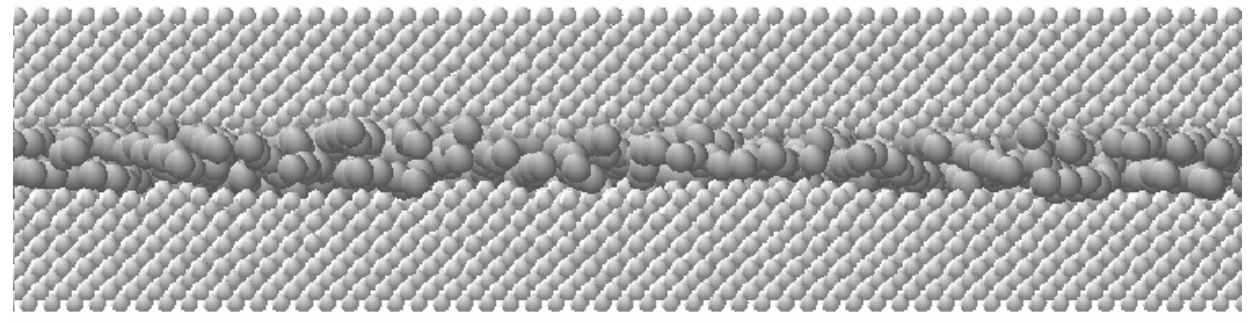

(b)

Fig. 9. 'Rough' surfaces used in the MD simulations. The surfaces were either non-adhesive or adhesive, and separated either by vacuum (a) or liquid hexadecane molecules (b). These simulations addressed both the lateral (shear, friction) forces [3] and the normal forces between the surfaces [4].

Turning now to the normal as opposed to the lateral or shear forces between rough surfaces (and the relationships between them), we have initiated a new series of experiments that look at surfaces of 'controlled' roughness rather than being simply randomly rough. These polymer surfaces are being prepared with asperities of known height distribution and surface coverage. We are particularly interested in seeing how the adhesion and friction forces between such surfaces differ from those between molecularly smooth surfaces. Our initial results confirm what 
has already been known, viz., that the elastic moduli of the surfaces (or the underlying material) must be considered in addition to the roughness. This is because the elastic modulus affects how the asperities deform when the two surfaces come into contact. For hard materials, or for polymers at temperatures well below their glass transition temperature $\left(T<T_{g}\right)$, the effective adhesion force or surface energy per unit area can be very low - often many orders of magnitude below the value for two ideal (molecularly smooth) surfaces - since it is determined by a few isolated asperities of low radii of curvature that do not deform when they come into contact (Fig. 10, top). In contrast, as illustrated in Fig. 10 (bottom), soft materials or surfaces with protruding surface groups can have adhesion and friction forces orders of magnitude higher than for ideal surfaces.

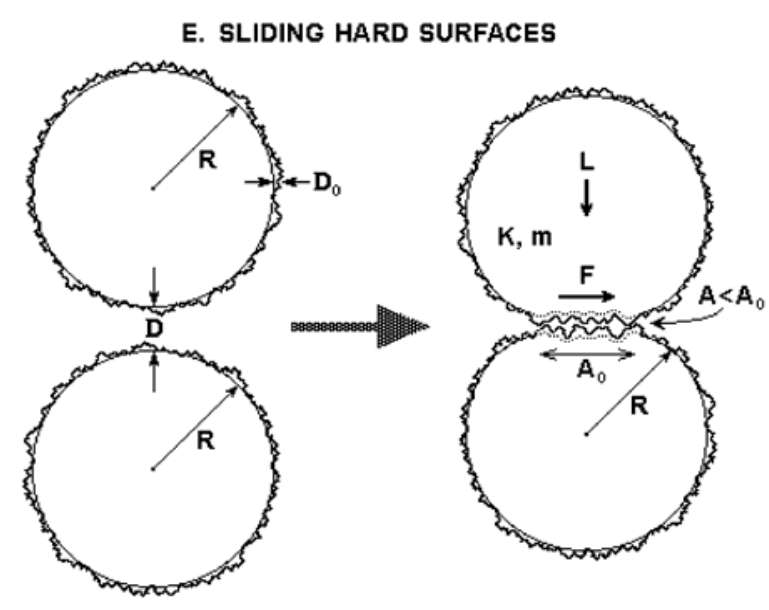

F. SLIDING VISCOELASTIC SURFACES

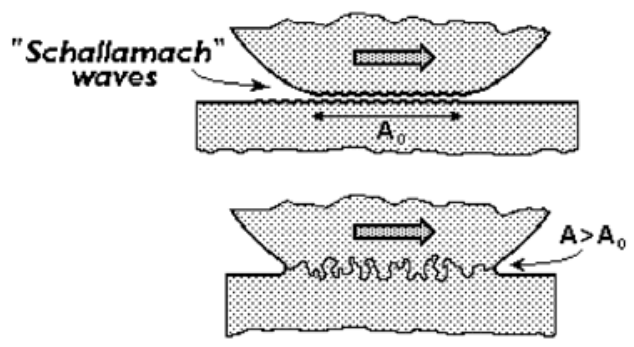

Fig. 10. Top: for hard material surfaces, or polymer surfaces below $\mathrm{T}_{\mathrm{g}}$, their 'real' contact area $\mathbf{A}$ is much less than the 'ideal' contact area $\mathbf{A}_{\mathbf{0}}$ for smooth surfaces, and so is their adhesion force. Bottom: for soft, ductile surfaces, or polymers at temperatures well above $T_{\mathrm{g}}$, we now find that $A>A_{0}$, and both the adhesion and friction forces are well above the ideal values. Our recent work suggests that the 'real' or effective contact area $\mathbf{A}$ of polymer materials is determined by the interdigitated chains within a thin interfacial layer, no more than about $1 \mathrm{~nm}$ thick. Rough but soft surfaces can also increase their effective contact area by elastically deforming.

We are currently investigating the quantitative relationships between roughness, surface elasticity/plasticity, and the adhesion and friction forces.

We are also investigating, both experimentally and theoretically, the normal force-distance function for rough surfaces (not just the adhesion force or energy), and have carried out a number of direct force-distance measurements on a number of different systems, including roughened silica and iron oxide, particle-covered, and rough custom-made polymer surfaces. Without exception, we find that that all these systems exhibit an exponentially repulsive force regime following the initial, weakly adhesive contact (determined by the touching of the outermost asperities). This is illustrated in Fig. 11 for two different surfaces in different environments (air and liquid). The exponential repulsion has been measured between materials that are hard or soft, and both in air and in liquids. It can be reversible or irreversible depending on the material. Experiments of the forces between polymer surfaces with tailor-made roughness are in progress. The origin of this apparently universal exponentially repulsive force law is a mystery. However, we have continued our collaboration with Dr Uzi Landman's group on this subject as well, and their MD simulations, which were carried out on the same rough surfaces as those that simulated the friction forces (Fig. 9) revealed an exponential repulsion very much as 
observed. This work is currently being written up as another joint experimental-theoretical paper [4]. A universal exponential repulsion has important implications for tribology, colloid science, powder technology and materials science. For example, the density or volume of granular materials have long been known to have a logarithmic dependence on the externally applied pressure (as found in the compaction stage during the processing of ceramic materials, for example). It is a simple matter to show that an exponentially repulsive force between the grains leads to a Density $\propto \log ($ Pressure $)$ relationship in a first approximation.
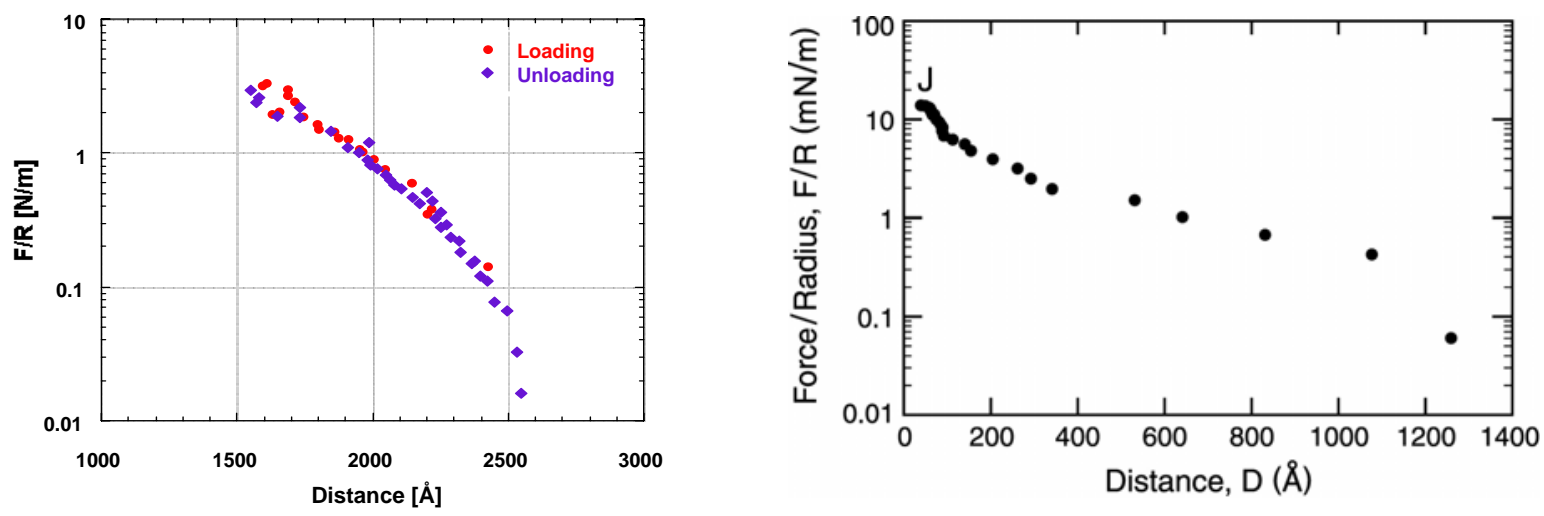

Fig. 11. Forces between different surfaces having different roughness, environments, and material compliance all show an exponentially repulsive regime over a significant range of distances. Left: reversible repulsion between rough and porous silica surfaces in air. Right: irreversible repulsion between rough iron oxide surfaces in water.

\section{Invited review article for Nature (on simplified theories of friction)}

There are three basic theoretical approaches for analyzing tribological systems: Molecular Dynamics (MD) simulations, rate-and-state models, and simple "minimalist" models that nevertheless include all the important features of MD simulations (which are the most rigorous). Figure 12 (top, left) illustrates one of the minimalist models developed by M. Urbakh and J. Klafter that can explain surprisingly complex behavior, including periodic and chaotic stick-slip and the non-Newtonian velocity dependence of the effective viscosity of thin lubricating films.
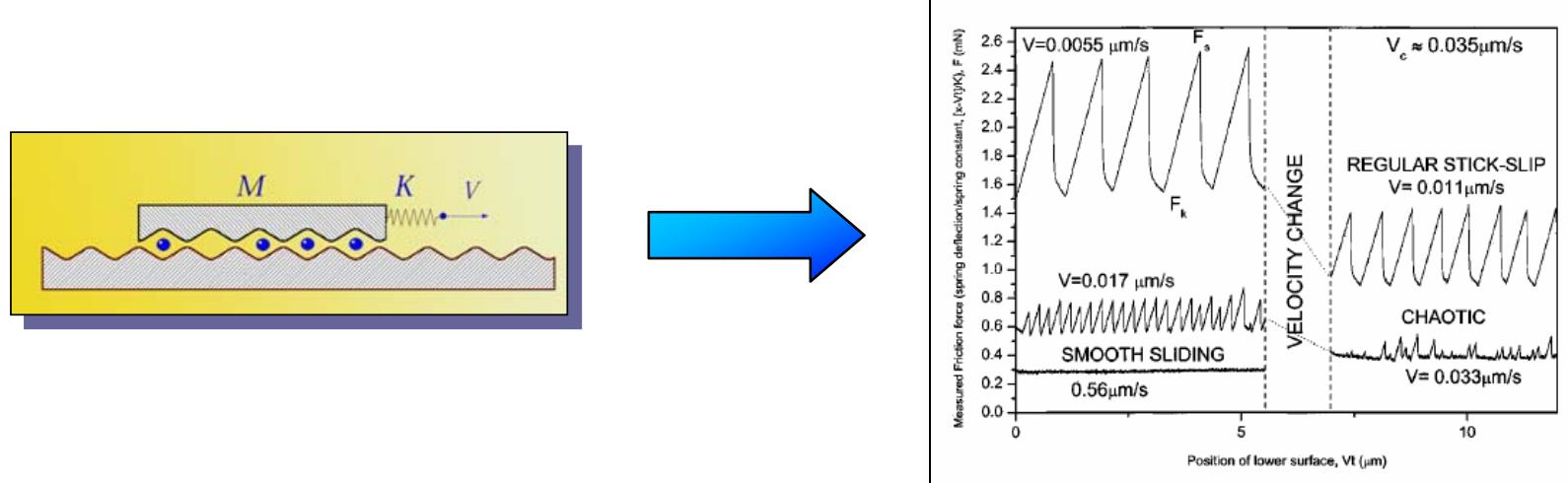

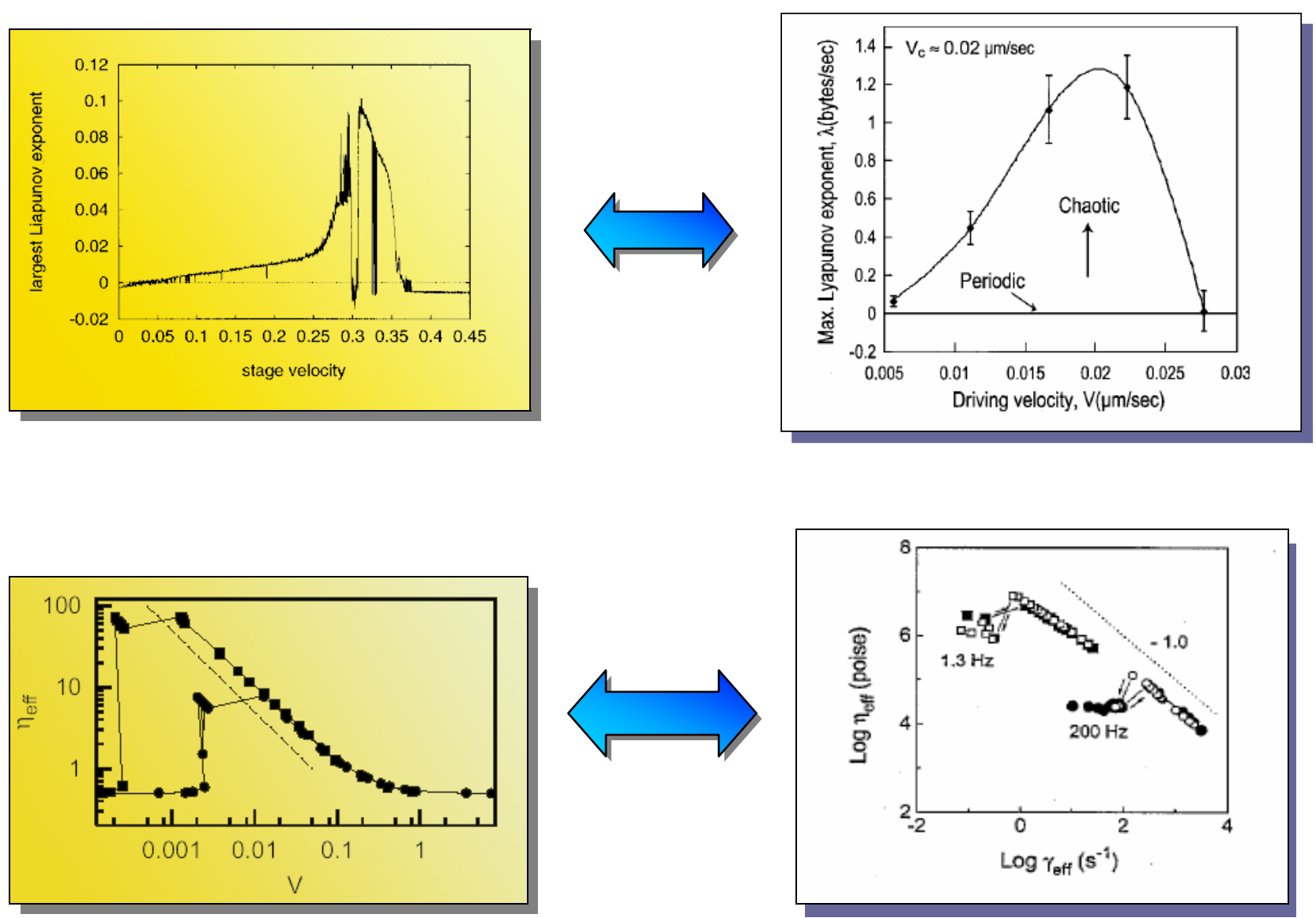

Fig. 12. Three examples, taken from Ref. [5], showing how the highly simplified model presented in the top left panel can explain highly complex tribological behavior (previously published experimental data shown on the right). An invited review article is being prepared for Nature [5], describing the three theoretical approaches for analyzing complex tribological systems and predicting their behavior.

\section{Measuring molecular organization in thin shearing interfacial films}

Preliminary results have been obtained using anisotropically light-absorbing dye molecules to study the ordering, including the alignment/orientation, of molecules in thin films as they are sheared. The results to date indicate that even after prolonged shearing, linear or anisotropic molecules do not become fully oriented along the sliding direction (or along any direction) even as the friction forces change dramatically with the shearing time until steady-state sliding is reached. This indicates that large tribological effects can occur when molecules change their ordering by only a small amount. This is consistent with some earlier MD simulations by Robbins et al., as well as our recent experimental results on polymer friction, described in Section 3. Studies on this interesting and potentially important phenomenon are continuing. The 'importance' of the phenomenon lies in the way we could better control frictional energy losses in the future once we understand which molecules or molecular groups are most involved. 


\section{Fundamental studies of crude oil-surface-water interactions and its relationship to reservoir wettability and oil recovery}

This work has now ended with the completion of an extensive series of experiments whose results, we believe, provide a fairly full understanding of the factors that determine crude oilwater-surface wettability, which is crucial for any oil-recovery process. The paper, which contains 17 figures, is due to appear soon [6]. The aim of our study was to directly measure the interactions between the various surfaces and interfaces (oil-water, oil-surface, asphaltenesurface, water-surface, etc.) in the presence of the third medium, and to establish how wetting phenomena such as contact angles, are determined. (This work was originally funded by Exxon and Mobile until they merged, further funding from Exxon-Mobile may materialize once their 'restructuring' is complete.)

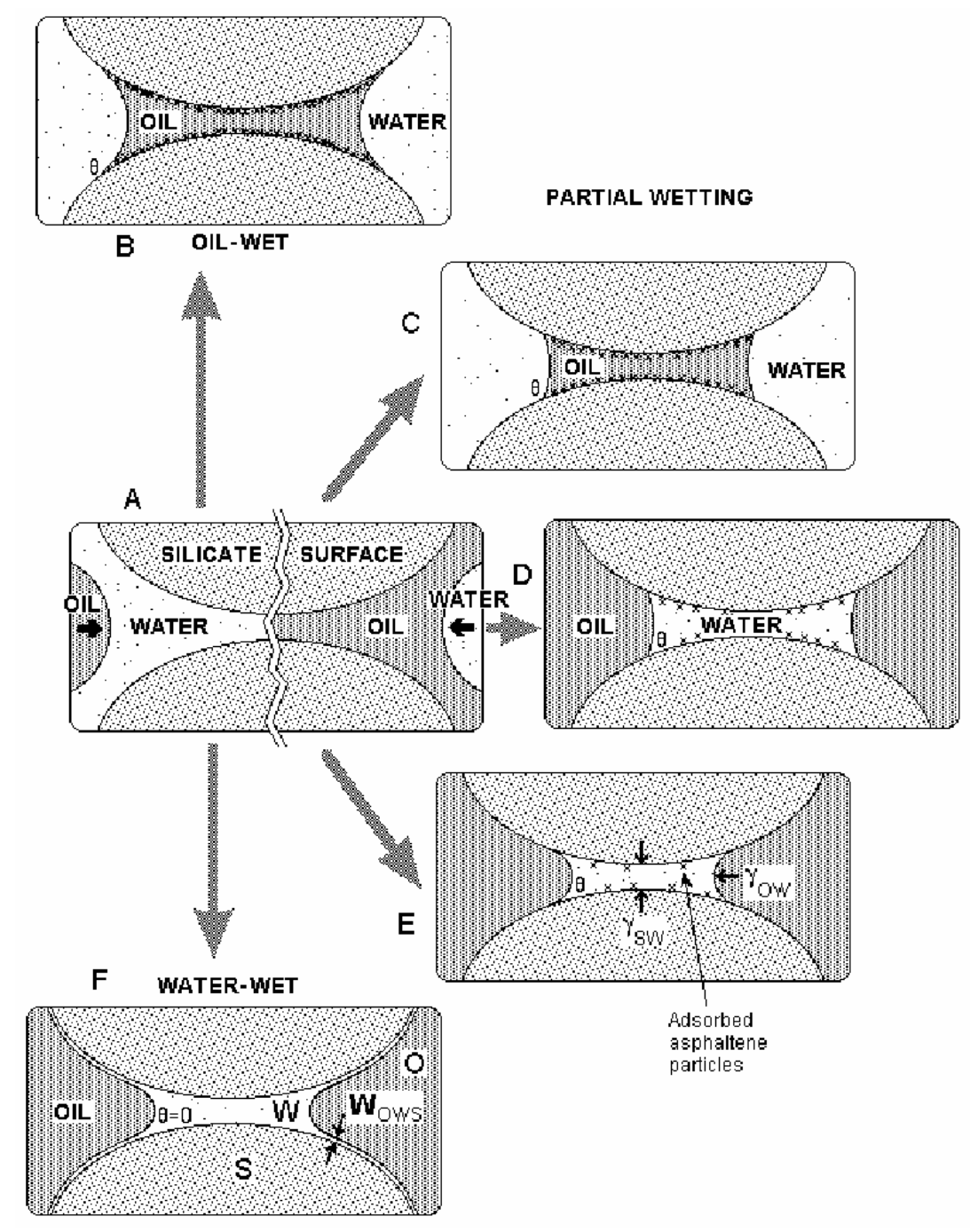

Fig. 13.

Equilibrium wetting configurations for decreasing water contact angles. Equilibrium can take a long time because of slow adsorption and surface diffusion of asphaltenes, as manifested by the differences in the advancing and receding contact angles, and in the slowly changing adsorbed layer thicknesses and corresponding surface forces as measured in the SFA. Figure taken from [6].

A Surface Forces Apparatus (SFA) was used to measure the adsorption on and the forces between molecularly smooth mica surfaces confining thin films of crude oil, brine or both, at different salinity and $\mathrm{pH}$. From contact angle measurements a 'wettability map' was established for the crude-oil system investigated showing the regions of different contact angles or 
'wettability' as a function of $\mathrm{pH}$ and ionic strength. This wettability map was found to be correlated with the independently measured surface forces and (mainly asphaltene) adsorption. The relative importance of different colloidal forces (van der Waals, electrostatic, etc.) on the observed wetting behavior was established, and in some cases quantitative agreement between measured contact angles and measured adhesion forces was obtained. We conclude that in addition to van der Waals and electrostatic forces (included in the DLVO theory), polymer-like interactions of adsorbed asphaltene layers are involved in the overall process. It was further established that the concept of an intrinsically oil-wet or water-wet surface or reservoir can not, in general, be correct: depending on the nature of the oil and brine, different species in a crude oil will adsorb on the mineral surfaces and at the oil/water interface, thereby determining the wetting behavior of a reservoir or rock.

\section{Research planned for 2004}

In addition to continuing or completing some of the seven projects described above we propose to initiate the following new studies, as envisaged in the original proposal for this grant period:

Using the recently completed and successfully tested three-dimensional or "XYZ” translation and detector stages for the SFA. Incidentally, JNI was recently awarded two patents for each of these devices or attachments, which can also be used in AFM experiments. The two patents are: (i) February, 2001 US Patent number 6,194,813 “Extended-range XYZ linear piezo-mechanical scanner for scanning-probe and surface force applications” and (ii)June, 2003 US Patent number 6,578,410 "Resistive cantilever spring for probe microscopy".

Using the new 'balance attachment' for the SFA to measure very long relaxation times and very weak forces.

\section{Miscellaneous information (conference presentations, awards, personnel)}

\section{Conference presentations}

Discussion Leader at the Gordon Research Conferences on Tribology, Roger Williams University, R.I., August 2003.

Keynote speaker at the annual meeting of the Society of Engineering Science, Penn. State, October 2002. Title of talk: "Equilibrium Versus Non-equilibrium Assembly of Nano-structures in Solution”.

Invited speaker at EUCHEM conference on Molecular Tribology, Sweden, December 2002, "Molecular mechanism associated with smooth to stick-slip sliding transitions".

Award Lecture at 2003 Adhesion Society Meeting, Myrtle Beach, South Carolina, February 2003. "Energy dissipation at the molecular and microscopic levels during dynamic adhesion processes". 
Invited talk at Gordon Research Conference on Polymers, Ventura, January 2003.

"Distinguishing between molecular, surface and bulk effects in the adhesion and friction of polymer surfaces”.

Invited talk at Mechanics of Material Interfaces Workshop, Research Triangle Park, N.C., May 2003, "Microscopic- and molecular-scale adhesion and friction processes”.

AFOSR Tribology Program Review, US Naval Academy, Annapolis, MD, August 2003.

"Relationship between the physical and electro-chemical interactions during tribological processes”.

Invited talk at the 50th AVS Symposium, Baltimore, November 2003. "Molecular and bulk material mechanisms of smooth and stick-slip sliding".

Invited paper for MRS 2003 Symposium P on "Dynamics in Small Confining Systems VII”, Boston, December 2003. "Inter-chain interactions and dynamics at shearing polymer interfaces”.

\section{Awards}

Prof. Jacob Israelachvili was the winner of this year's Adhesion Society's Award for Excellence in Adhesion Science, sponsored by 3M. The award was given at a special conference session in his honor at the Annual Meeting of the Adhesion Society in Myrtle Beach, February, 2003.

\section{Graduate students and post-docs fully or partially funded by this DOE grant}

Name

Status

Completion/graduation date

Grad students (MS, PhD)

Norma Alcantar

PhD student

End of 2002

Nianhuan Chen

PhD student

End of 2003

Qi Lin

PhD student

Ongoing

Mustafa Akbulut

PhD student

Ongoing

\section{Post docs}

Dr Norma Alcantar

Post-doc

$1 / 2003$ to $12 / 20 / 03^{*}$

Dr Delphine Gourdon

Post-doc

Ongoing

Dr Nobuo Maeda

Post-doc

Ongoing

* Dr Alcantar is taking up a position of Assistant Professor at the University of Florida (Tampa) in December 2003.

Tech support

Greg Carver

Development Engineer Ongoing 